\title{
Axiom of Choice in nonstandard set theory
}

\author{
KAREL HRBACEK
}

\begin{abstract}
We verify that the best-known nonstandard set theories: IST, BST, and HST, with the Axiom of Choice deleted, are conservative extensions of $\mathbf{Z F}+$ Boolean Prime Ideal Theorem.

2010 Mathematics Subject Classification 26E35 (primary); 03E25, 03E70, $03 \mathrm{H} 05$ (secondary)

Keywords: axiom of choice, nonstandard analysis, nonstandard set theory, IST, BST, HST
\end{abstract}

Nonstandard set theories typically postulate validity of the axioms of $\mathbf{Z F C}$, including the Axiom of Choice, in the standard (or internal) universe. They also postulate some versions of Transfer, Standardization and Idealization. An easy argument shows that the last three principles, together with $\mathbf{Z F}$ alone, imply the Boolean Prime Ideal Theorem (BPI):

Let $\mathcal{F}$ be a standard filter over a standard set $S=\bigcup \mathcal{F}$. By Idealization, there is an (internal) element $x \in S$ such that $x \in X$ for all standard $X \in \mathcal{F}$. By Standardization, there is a standard $\mathcal{U} \subseteq \mathcal{P}(S)$ such that $X \in \mathcal{U} \leftrightarrow x \in X$, for all standard $X \in \mathcal{P}(S)$. Trivially, $\mathcal{U}$ is a standard ultrafilter and $\mathcal{F} \subseteq \mathcal{U}$. By Transfer, for every filter $\mathcal{F}$ there is an ultrafilter $\mathcal{U}$ such that $\mathcal{F} \subseteq \mathcal{U}$. This statement ("Ultrafilter Theorem") is one of the forms equivalent to BPI; see Jech [5, Theorem 2.2].

The purpose of this note is to point out that this is the strongest possible result. We use the monograph of Kanovei and Reeken [7] as a general reference to nonstandard set theories. The best known among these is Nelson's IST, so we begin our considerations with IST.

Let IST $^{-}$be IST with the Axiom of Choice deleted. The argument above shows that IST $^{-} \vdash$ BPI.

Theorem 1 IST $^{-}$is a conservative extension of $\mathbf{Z F}+B P I$.

The analogous result for IST, to wit, that IST is a conservative extension of ZFC, is proved using ultrapowers. While BPI guarantees existence of many ultrafilters, it does 
not guarantee validity of Łos̀ Theorem for the corresponding ultrapowers, a property needed to obtain Transfer. In fact, "BPI + Łos̀ Theorem for all ultrapowers" implies the Axiom of Choice (in $\mathbf{Z F}$ ); see Howard and Rubin [2]. However, we can replace the ultrapower construction by one based on the Compactness Theorem.

The proof of Theorem 1 is a result of a series of easy observations. We work in $\mathbf{Z F}$, unless stated otherwise.

The Compactness Theorem is one of the equivalents of BPI given in [5, Theorem 2.2]. Another equivalent stated there is the Consistency Theorem:

For every binary mess $M$ on a set $S$, there exists a function $f$ on $S$ which is consistent with $M$.

We recall that a binary mess $M$ on $S$ is a set of functions defined on finite subsets of $S$ and with values in $\{0,1\}$, closed under restrictions, and such that for each finite $P \subseteq S$ there is $t \in M$ with $\operatorname{dom} t=P$.

A function $f: S \rightarrow\{0,1\}$ is consistent with $M$ if $f \mid P \in M$ for every finite $P \subseteq S$.

We need the following simple observation.

\section{Proposition 2 BPI implies the Uniform Consistency Principle:}

Let $\left\langle M_{i}: i \in I\right\rangle$ be a system of binary messes, with $I$ an arbitrary set. Then there is a system of functions $\left\langle f_{i}: i \in I\right\rangle$ such that $f_{i}$ is consistent with $M_{i}$, for each $i \in I$.

Proof Each $M_{i}$ is a binary mess on $S_{i}=\bigcup\left\{\operatorname{dom} t: t \in M_{i}\right\}$. Wlog we can assume that the sets $S_{i}$ are mutually disjoint. Let $S=\bigcup_{i \in I} S_{i}$ and let $t \in M$ iff $\operatorname{dom} t \subseteq S$ is finite and $t \mid S_{i} \in M_{i}$ for all $i \in I$. Trivially, $M$ is a mess. If $f$ is consistent with $M$, then $\left\langle f \mid S_{i}: i \in I\right\rangle$ is a system of functions, and each $f \mid S_{i}$ is consistent with $M_{i}$.

An examination of the proof of [5, Theorem 2.2] shows that the Uniform Consistency Principle [hence, BPI] implies uniform versions of the other forms given there. For example, BPI implies

Uniform BPI: Let $\left\langle B_{i}: i \in I\right\rangle$ be a system of Boolean algebras. Then there is a system $\left\langle J_{i}: i \in I\right\rangle$ such that each $J_{i}$ is a prime ideal on $B_{i}$.

Importantly for our purposes, it also implies

Uniform Compactness Theorem: Let $\left\langle\Sigma_{i}: i \in I\right\rangle$ be a system of consistent theories. Then there is a system of structures $\left\langle\mathfrak{M}_{i}: i \in I\right\rangle$ such that $\mathfrak{M}_{i} \vDash \Sigma_{i}$, for all $i \in I$. 
Definition Let $*: V \rightarrow{ }^{*} V$ be an elementary $\in$-embedding of an $\in$-structure $\mathbf{v}=$ $\langle V, \varepsilon\rangle$ into an $\in$-structure ${ }^{*} \mathbf{v}=\left\langle{ }^{*} V,{ }^{*} \varepsilon\right\rangle$, with $V,{ }^{*} V$ sets; see [7, page 138]. We say that ${ }^{*} \mathbf{v}$ is an enlargement of $\mathbf{v}$ via $*$ if for every $\in$-formula $\Phi\left(x, y, z_{1}, \ldots, z_{k}\right)$, all $p_{1}, \ldots, p_{k} \in V$, and all $S \subseteq V$ : If for all finite $\left\{a_{1}, \ldots, a_{n}\right\} \subseteq S$ there is $b \in V$ such that $\mathbf{v} \vDash \bigwedge_{i=1}^{n} \Phi\left(a_{i}, b, p_{1}, \ldots, p_{k}\right)$, then there is $b \in{ }^{*} V$ such that, for all $a \in S$, ${ }^{*} \mathbf{v} \vDash \Phi\left({ }^{*} a, b,{ }^{*} p_{1}, \ldots,{ }^{*} p_{k}\right)$.

Proposition $3(\mathbf{Z F}+\mathrm{BPI}) \quad$ Every $\in$-structure $\mathbf{v}$ has an enlargement.

Proof Start with the elementary diagram $T_{0}$ of $\mathbf{v}$ (Chang and Keisler [1, Chapter 3]). For every $\in$-formula $\Phi$, every $p_{1}, \ldots, p_{k} \in V$, and every $S \subseteq V$ for which the finite satisfiability condition above holds, add to the language of $T_{0}$ a new constant $c$, and add to $T_{0}$ the formulas $\Phi\left(\underline{a}, c, p_{1}, \ldots, p_{k}\right)$, for all $a \in S$. The resulting theory $T_{1}$ is consistent, and by the Compactness Theorem it has a model $\mathfrak{M}$. Let ${ }^{*} \mathbf{v}$ be the reduct of $\mathfrak{M}$ to the $\in$-language, and $*: a \mapsto \underline{a}^{\mathfrak{M}}$ for $a \in V$; then $*$ is an elementary $\in$-embedding and ${ }^{*} \mathbf{v}$ is an enlargement of $\mathbf{v}$ via $*$.

From Uniform Compactness Theorem and the proof of Proposition 3 one obtains:

Corollary $4(\mathbf{Z F}+\mathrm{BPI})$ Let $\left\langle\mathbf{v}_{i}: i \in I\right\rangle$ be a system of $\in$-structures. Then there are systems $\left\langle{ }^{*} \mathbf{v}_{i}: i \in I\right\rangle$ and $\left\langle *_{i}: i \in I\right\rangle$ such that each ${ }^{*} \mathbf{v}_{i}$ is an enlargement of $\mathbf{v}_{i}$ via $*_{i}$.

Observation If an $\in$-structure $\mathbf{v}$ has von Neumann rank $\xi$, then the enlargement ${ }^{*} \mathbf{v}$ constructed in the proof of Proposition 3 has rank only a few levels above $\xi$; certainly $\mathbf{v} \in \mathbf{V}_{\xi+1}$ implies ${ }^{*} \mathbf{v} \in \mathbf{V}_{\xi+10}$. [We can use something like $\langle a, \mathbf{v}\rangle$ as the name $\underline{a}$ for $a \in V$, and $\left\langle\Phi, p_{1}, \ldots, p_{k}, S, \mathbf{v}\right\rangle$ as the new constant $c ;{ }^{*} V$ is constructed from equivalence classes of names; ${ }^{*} \varepsilon$ is a collection of ordered pairs of elements of ${ }^{*} V$; ${ }^{*} \mathbf{v}=\left\langle{ }^{*} V,{ }^{*} \varepsilon\right\rangle$.] Hence if $\theta>\omega$ is a limit ordinal, then $\mathbf{v} \in \mathbf{V}_{\theta}$ implies ${ }^{*} \mathbf{v} \in \mathbf{V}_{\theta}$.

Let $\mathbf{Z F}$ denote the fragment of $\mathbf{Z F}$ where only the first $\ell$ instances of Replacement are postulated; similarly for $\mathbf{I S T}_{\ell}^{-}$. (Note that Collection is not considered part of ZF.)

\section{Proof of Theorem 1}

Let $\varphi$ be a sentence in the $\in$-language. We assume that $\mathbf{Z F}+\mathrm{BPI}+\varphi$ is consistent and show that $\mathbf{I S T}^{-}+\varphi$ is consistent. It suffices to show that $\mathbf{I S T}_{\ell}^{-}+\varphi$ is consistent, for every fixed $\ell$.

We work in $\mathbf{Z F}+$ BPI $+\varphi$. The Reflection Principle [7, Theorem 1.5.4(i)] is provable in $\mathbf{Z F}$. Hence there is an ordinal $\gamma$ such that $\mathbf{v}=\left\langle\mathbf{V}_{\gamma}, \in \uparrow \mathbf{V}_{\gamma}\right\rangle \vDash \mathbf{Z F}_{\ell}+$ BPI $+\varphi$. We 
fix a limit ordinal $\theta$ such that $\mathbf{v} \in \mathbf{V}_{\theta}$. Let $\left\langle\mathbf{v}_{i}: i \in I\right\rangle$ be a system that enumerates all $\in$-structures in $\mathbf{V}_{\theta}$ in a one-one way (we can use the structures themselves as indices). Using Corollary 4 and Observation, we define:

$\mathbf{v}_{0}=\mathbf{v}, \mathbf{v}_{n+1}={ }^{*} \mathbf{v}_{i}, *_{n, n+1}=*_{i}$ where $\mathbf{v}_{i}=\mathbf{v}_{n}, *_{m, n+1}=*_{n, n+1} \circ *_{m, n}$ for $m<n$ (below, we omit the subscripts when they should be clear from the context).

Let ${ }^{\star} \mathbf{v}=\left\langle{ }^{\star} V,{ }^{\star} \varepsilon\right\rangle$ be the direct limit of this system, with $\star_{n}: \mathbf{v}_{n} \rightarrow{ }^{\star} \mathbf{v}$ the canonical embeddings. As $\star=\star_{0}: \mathbf{V}_{\gamma} \rightarrow{ }^{\star} V$ is elementary, ${ }^{\star} \mathbf{v} \vDash \mathbf{Z F}_{\ell}+$ BPI $+\varphi$. Let ${ }^{\star} \mathbf{s t}=\left\{{ }^{\star} X: X \in \mathbf{V}_{\gamma}\right\}$, so $\left\langle{ }^{\star} \mathbf{s t},{ }^{\star} \varepsilon\left\lceil{ }^{\star} \mathbf{s t}\right\rangle\right.$ is isomorphic to $\left\langle\mathbf{V}_{\gamma}, \in \uparrow \mathbf{V}_{\gamma}\right\rangle$. We consider the structure $\mathfrak{M}=\left\langle{ }^{\star} V,{ }^{\star} \varepsilon,{ }^{\star} \mathbf{s t}\right\rangle$ and show that it satisfies $\mathbf{I S T}_{\ell}^{-}+\varphi$.

As noted above, $\mathfrak{M}$ satisfies $\mathbf{Z F} \mathbf{F}_{\ell}+\mathrm{BPI}+\varphi$. The elementarity of the embedding $\star$ also implies that $\mathfrak{M}$ satisfies Transfer. If $\Phi(x)$ is a formula of the st- $\in$-language (with parameters from ${ }^{\star} V$ ) and ${ }^{\star} X \in{ }^{\star}$ st, let $Y=\left\{x \in X: \mathfrak{M} \vDash \Phi\left({ }^{\star} x\right)\right\}$. As $Y \in \mathbf{V}_{\gamma}$, we have $\mathfrak{M} \vDash \mathbf{s t}\left({ }^{\star} Y\right) \wedge\left(\forall^{\text {st }} y\right)\left(y \in{ }^{\star} Y \leftrightarrow y \in{ }^{\star} X \wedge \Phi(y)\right)$, so Standardization holds in $\mathfrak{M}$.

Finally, we note that standard finite sets in $\mathfrak{M}$ are precisely the sets of the form ${ }^{\star} A$ where $A \in \mathbf{V}_{\gamma}$ is finite. Let an $\in$-formula $\Phi\left(x, y, z_{1}, \ldots, z_{k}\right)$ and $p_{1}, \ldots, p_{k} \in{ }^{\star} V$ be such that, for every finite $A \in \mathbf{V}_{\gamma}, \mathfrak{M} \vDash(\exists b)\left(\forall a \in{ }^{\star} A\right) \Phi\left(a, b, p_{1}, \ldots, p_{k}\right)$. Then there exist $\mathbf{v}_{n}=\left\langle V_{n}, \varepsilon_{n}\right\rangle$ and $q_{1}, \ldots, q_{k} \in V_{n}$ such that ${ }^{\star} q_{i}=p_{i}$ for $i \leq k$. By elementarity of $\star_{n}$, for every finite $A=\left\{a_{1}, \ldots, a_{m}\right\} \subseteq \mathbf{V}_{\gamma}$ there is $b \in V_{n}$ such that $\mathbf{v}_{n} \vDash \bigwedge_{i=1}^{m} \varphi\left({ }^{*} a_{i}, b, q_{1}, \ldots, q_{k}\right)$. As $\mathbf{v}_{n+1}=\left\langle V_{n+1}, \varepsilon_{n+1}\right\rangle$ is an enlargement of $\mathbf{v}_{n}$ (via $\left.*_{n, n+1}\right)$, there is $b \in V_{n+1}$ such that $\mathbf{v}_{n+1} \vDash \Phi\left({ }^{*} a, b,{ }^{*} q_{1}, \ldots,{ }^{*} q_{k}\right)$ for all $a \in \mathbf{V}_{\gamma}$. By elementarity of the embedding $\star_{n+1}$ then $\mathfrak{M} \vDash \Phi\left({ }^{\star} a,{ }^{\star} b, p_{1}, \ldots, p_{k}\right)$ for all $a \in \mathbf{V}_{\gamma}$. This establishes Idealization in $\mathfrak{M}$, and completes the proof of Theorem 1.

Kanovei [6] formulated BST, a modification of IST with better metamathematical behavior. In particular, BST is precisely the "internal part" of the nonstandard set theories HST and NST, introduced essentially in [3]. It is well known that BST has an interpretation in IST [7, Theorem 3.4.5(i)]; the sets of BST are interpreted as bounded sets of IST [where $x$ is bounded iff $x \in X$ for some standard $X$ ]. The proof goes through even in the absence of the Axiom of Choice, and thus establishes that BST ${ }^{-}$ (ie, BST with the Axiom of Choice deleted) is interpretable in IST $^{-}$. As the standard sets of BST $^{-}$in this interpretation are precisely the standard sets of IST $^{-}$, we have the following consequence of Theorem 1.

Corollary 5 BST $^{-}$is a conservative extension of $\mathbf{Z F}+B P I$.

However, BST has a much stronger property: It has a standard core interpretation in ZFC [7, Definition 4.1.3 and Theorem 4.3.17]. It is an open question whether BST ${ }^{-}$ 
has a standard core interpretation in $\mathbf{Z F}+$ BPI, or even just whether every model of $\mathbf{Z F}$ + BPI can be extended to a model of BST $^{-}$.

Among nonstandard set theories that allow external sets, HST is distinguished-among other properties-by having an internal core interpretation in BST; see [7, Definition 5.1.2 and Corollary 5.1.5]. We next consider the role of the Axiom of Choice in HST.

The Axiom of Choice (AC) appears in HST in three places. It is postulated to hold in the standard universe $\left(\mathrm{AC}^{\text {st }} ;[7,1.1 \mathrm{c}]\right.$ ), and Standard Size Choice (which implies $\mathrm{AC}^{\text {st }}$ ) and Dependent Choice (DC) are postulated as axioms about sets in the external universe [7, 1.1f]. We let HST ${ }^{-}$be HST with these three axioms deleted.

Theorem 6 HST $^{-}$is a conservative extension of $\mathbf{Z F}+B P I$.

For the proof, we verify that the argument of [7], showing that HST has an internal core interpretation in BST, can be carried out in $\mathbf{B S T}^{-}$, with the exception of the three deleted choice axioms. This argument is structured roughly into three steps.

Step 1 In Chapter 3 of [7], the authors deduce some important consequences of BST. We list below those results whose proof in [7] uses AC, and discuss their status in BST $^{-}$.

[7, Lemma 3.1.14]: (BIST) Let $\Phi(x)$ be an $\in$-formula with standard parameters and a parameter $w \in W$, where $W$ is standard. If there is a unique set $x$ with $\Phi(x)$ then this $x$ belongs to $\mathbb{S}[w]$. If $w$ is standard then so is $x$.

This lemma remains valid in $\mathbf{B S T}^{-}$(even BIST $^{-}$).

Proof Let $\Phi(x, w)$ be an $\in$-formula with standard parameters and a parameter $w \in W$, where $W$ is standard, such that there is a unique $x$ with the property $\Phi(x, w)$. Using Regularity of $\mathbf{Z F}$ and Inner Transfer, we define a standard function $F$ for $w^{\prime} \in W$ by $F\left(w^{\prime}\right)=\left\{x: \Phi\left(x, w^{\prime}\right) \wedge\left(\forall x^{\prime}\right)\left(\Phi\left(x^{\prime}, w^{\prime}\right) \rightarrow \operatorname{rank} x \leq \operatorname{rank} x^{\prime}\right)\right\}$ [where rank $x$ is the rank of $x$ in the von Neumann cumulative hierarchy $\left\langle\mathbf{V}_{\xi}: \xi \in \mathbf{O r d}\right\rangle$ ]. By [7, Definition 3.1.13], $F(w) \in \mathbb{S}[w]$. But $F(w)=\{x\}$ for the unique $x$ with the property $\Phi(x, w)$; so also $x \in \mathbb{S}[w]$.

We remark that in $\mathbf{B S T}^{-}$the class $\mathbb{S}[w]=\{f(w): f$ is a standard function and $w \in$ $\operatorname{dom} f\}$ need not be an $\in$-elementary substructure of the internal universe $\mathbb{I}$ (compare [7, Theorem 6.1.3(i)]). Indeed, every ultrapower of the standard universe modulo a standard ultrafilter is canonically isomorphic to some $\langle\mathbb{S}[w], \in\rangle$ (essentially [7, Proposition 6.1.9], which remains valid in $\mathbf{B S T}^{-}$), so if $\mathbb{S}[w] \prec \mathbb{I}$ for all $w$, then $\mathbb{S} \prec \mathbb{S}[w]$ 
for all $w$, and Łos̀ Theorem holds for all ultrapowers. As noted above, this implies the full Axiom of Choice.

[7, Lemma 3.1.16]: (BIST) Let $\Phi(x, y)$ be a $\mathbf{s t -} \in-$-formula with any parameters. Then, for any standard sets $A, B$, we have:

$$
\forall^{\text {st }} a \in A \exists^{\text {st }} b \in B \Phi(a, b) \Longleftrightarrow \exists^{\text {st }} f \in B^{A} \forall^{\text {st }} a \in A \Phi(a, f(a)) .
$$

$\mathrm{AC}$ is essential for the validity of this lemma. However, this lemma is used only in the chain of arguments leading to the proof that Standard Size Choice holds in the interpretation of HST in BST [proof of Map-Standardization and Inner S. S. Choice in Theorem 3.2.11, Standard Size Choice for EEST in Theorem 5.2.14, and the verification of Standard Size Choice for HST on page 207].

We remark that the step of replacing the quantifier combination $\forall^{\text {st }} a \exists^{\text {st }} b$ with $\exists^{\text {st }} f \forall^{\text {st }} a$ in Nelson's original version of the Reduction Algorithm [8] is based on this lemma, and hence is unavailable in $\mathbf{B S T}^{-}$. However, the version of the Reduction Algorithm in [7, Theorem 3.2.3], due to Andreev, replaces $\forall^{\text {st }} a \exists^{\text {st }} b$ with $\exists^{\text {st }} U \forall^{\text {st }} x \in U$ (where $U$ ranges over ultrafilters) and is valid in $\mathbf{B S T}^{-}$.

[7, Lemma 3.2.7]: (BST) Assume that $\varphi(a, b, x)$ is a parameter-free $\in$-formula. For any standard set $X$ there exist standard sets $A$ and $B$ of cardinality resp. $\leq 2^{\kappa}$ and $\leq 2^{2^{\kappa}}$, where $\kappa=\operatorname{card} X$, such that for all $x \in X$ :

$\exists^{\text {st }} a \forall^{\text {st }} b \varphi(a, b, x) \Longleftrightarrow \exists^{\text {st }} a \in A \forall^{\text {st }} b \varphi(a, b, x) \Longleftrightarrow \exists^{\text {st }} a \in A \forall^{\text {st }} b \in B \varphi(a, b, x)$.

The lemma remains valid in BST $^{-}$if the cardinality estimates $\left(|A| \leq 2^{\kappa},|B| \leq 2^{2^{\kappa}}\right)$ are omitted. The estimates are not needed to carry out the argument; in particular, Inner Collection holds in BST $^{-}$(Theorem 3.2.8).

[7, Theorem 3.2.11]: (BST) Map-Standardization, Uniqueness, Inner S. S. Choice (hence Inner Extension), and Inner Dependent Choice hold.

The proofs of Map-Standardization and Inner S. S. Choice use AC, and Inner Dependent Choice uses DC; these principles cannot be proved in $\mathbf{B S T}^{-}$. But, as pointed out above, they are used in the argument only to establish Standard Size Choice and Dependent Choice, respectively. The proof of Uniqueness goes through in BST ${ }^{-}$. Inner Extension is crucial to establishing Saturation in the final interpretation. In Theorem 3.2.11 it is a consequence of Inner S. S. Choice. We give below a derivation in $\mathbf{B S T}^{-}$.

\section{Proof of Inner Extension:}

For any standard set $X$ there is a function $f$ defined on $X$ such that

$$
\left(\forall^{\text {st }} x \in X\right)((\exists ! y) \Phi(x, y) \rightarrow \Phi(x, f(x)) .
$$


By [7, Theorem 3.2.8 and Exercise 3.1.7(iii)], there is a standard set $Y$ such that $\left(\forall^{\mathbf{s t}} x \in\right.$ $X)((\exists y) \Phi(x, y) \rightarrow(\exists y \in Y) \Phi(x, y))$. Let $\widetilde{y}(x)$ denote this unique $y \in Y$, if it exists $(\widetilde{y}$ of course need not be a function-it is external). We may assume $\Phi$ has a single internal parameter $p$, where $p$ belongs to a standard set $P$. By Uniqueness, for each standard $x \in X$ we have $\widetilde{y}(x) \in \mathbb{S}[\langle x, p\rangle]=\mathbb{S}[p]$. Hence $\left(\forall^{\text {st }} x\right)\left(\exists^{\text {st }} g\right)$ [ $g$ is a function with $p \in$ $\operatorname{dom} g$ and $g(p)=\langle x, \tilde{y}(x)\rangle]$; wlog $\operatorname{dom} g=P$ and $\operatorname{ran} g \subseteq X \times Y$. We now let $G={ }^{s}\left\{g \in \mathbb{S}: g \in(X \times Y)^{P} \wedge\left(\exists^{\text {st }} x \in X\right)(\exists y \in Y)[g(p)=\langle x, y\rangle \wedge \Phi(x, y)]\right\}$. Thus $G$ is a standard set that "indexes" the graph of the "external function" $\tilde{y}$. We have $\left(\forall{ }^{\text {st fin }} G_{0} \subseteq G\right)(\exists f)\left(\forall g \in G_{0}\right)[\operatorname{dom} f \subseteq X \wedge(\forall x, y)(g(p)=\langle x, y\rangle \rightarrow f(x)=y)]$. We can define such $f$ by the condition $f(x)=y$ iff $\left(\exists g \in G_{0}\right)(g(p)=\langle x, y\rangle)$. Note that, for $g_{1}, g_{2} \in G_{0}, g_{1}(p)=\langle x, y\rangle, g_{2}(p)=\left\langle x, y^{\prime}\right\rangle$ imply (because $g_{1}, g_{2}$ are standard elements of $G$ ) that $x$ is standard $\wedge \Phi(x, y) \wedge \Phi\left(x, y^{\prime}\right)$, hence $y=y^{\prime}$, so $f$ is a welldefined (finite) function.

By Idealization, there exists $f$, a function defined on a subset of $X$, such that $\left(\forall^{\text {st }} g \in\right.$ $G)(\forall x, y)(g(p)=\langle x, y\rangle \rightarrow f(x)=y)$. For every standard $x \in X,\langle x, \tilde{y}(x)\rangle=g(p)$ for some standard $g \in G$, so we get $f(x)=\tilde{y}(x)$, for all standard $x$. We extend $f$ to a function defined on $X$ by letting $f(x)=0$ where previously undefined.

The key Theorem 3.2.16, establishing a parametrization of st- $\in$-definable "external sets," is now seen to hold in $\mathbf{B S T}^{-}$.

Step 2 EEST $^{-}$and its interpretation in BST $^{-}$.

Let EEST ${ }^{-}$be the theory EEST [7, Definition 5.1.3] with $\mathrm{AC}^{\text {st }}$ deleted. [7, Theorem 5.2.6] then provides an internal core interpretation of EEST ${ }^{-}$in $\mathbf{B S T}^{-}$. The properties of EEST from Section 5.2 carry over to EEST ${ }^{-}$, with the exception of the Standard Size Choice in Theorem 5.2.14, and Exercise 5.2.19 (the latter uses Inner Dependent Choice). However, Theorem 5.2.14 for a weaker version of Standard Size Choice does hold:

Extension For any set $X$ there exists an internal function $F$ such that

$$
\left(\forall^{\text {st }} x \in X\right)\left[\left(\exists !^{\text {int }} y\right) \Phi(x, y) \rightarrow \Phi(x, F(x))\right]
$$

[because the Inner Extension case in Theorem 3.2.11 holds], and this is all that is needed to prove Theorem 5.2.18 [Saturation].

Step 3 Assembling of external sets in HST $^{-}$and an interpretation of HST $^{-}$in EEST $^{-}$.

The only issue in Section 5.3 is the use of DC in the proof of Lemma 5.3.6, but this can be eliminated by arguing directly from Regularity over $\mathbb{I}$ : Given $X \subseteq T_{*}, X \neq \varnothing$, let 
$S=\left\{y: y=y_{i}\right.$ for some $\left.\left\langle y_{0}, \ldots, y_{i}, \ldots, y_{n}\right\rangle=t \in X\right\}$. By Regularity over $\mathbb{I}$ there is $y \in S$ such that $y \cap S \subseteq \mathbb{I}$. If $y=y_{i}$ as above, then either $y_{i}$ is internal and $i=n$, or $y_{i+1}$ is internal and $i+1=n$. In either case, $t \in \operatorname{Max} T_{*_{x}}$.

The construction of an interpretation of HST in EEST and the verification of the HST axioms in [7, Section 5.4] can be carried out in EEST $^{-}$without changes, except for Standard Size Choice and Dependent Choice. The latter follows if one has Exercise 5.2.19, ie, if one works in BST $^{-}+$DC. This concludes the proof of Theorem 6 , and also establishes the following corollary.

Corollary 7 HST $^{-}+$Dependent Choice is a conservative extension of $\mathbf{Z F}+B P I+$ $D C$.

We note that $\mathbf{Z F}+$ BPI + DC does not imply AC; see [5].

Other nonstandard set theories of external sets have been proposed; in particular NST and KST [7, Chapter 8]. Models of these theories are constructed, roughly speaking, as some kind of cumulative hierarchy over a suitable model of BST or IST. It is easy to start instead with a suitable model of $\mathbf{B S T}^{-}$or $\mathbf{I S T}^{-}$, and construct a cumulative hierarchy that satisfies all the axioms of NST or KST except Standardization and Saturation (and of course the (internal) Axiom of Choice). It is an open question whether the appropriate versions of Standardization and Saturation can simultaneously be satisfied.

There are also internal theories with many levels of standardness, such as RIST of Péraire [9] and GRIST proposed by the author in [4]. It is likely that these theories, without Choice, are conservative extensions of $\mathbf{Z F}+$ BPI, but the verification of this conjecture would require an inordinate amount of technical work.

\section{References}

[1] C C Chang and H J Keisler, Model Theory, 3rd edition, North-Holland Publ. Co., 1990.

[2] P Howard and JE Rubin, Consequences of the Axiom of Choice, Math. Surveys and Monographs, 59, Amer. Math. Society, Providence, RI, 1998, viii+ 432 pages.

[3] K Hrbacek, Axiomatic foundations for nonstandard analysis, Fundamenta Mathematicae 98 (1978), 1 - 19; abstract in J. Symbolic Logic 41 (1976), 285.

[4] K Hrbacek, Relative set theory: Internal view, Journal of Logic and Analysis 1:8 (2009), 1-108; doi: 10.4115/jla.2009.1.8.

[5] T J Jech, The Axiom of Choice, North-Holland Publ. Co., Amsterdam, 1973. 
[6] V Kanovei, Undecidable hypotheses in Edward Nelson's Internal Set Theory, Russian Mathematical Surveys 46 (1991), 1 - 54.

[7] V Kanovei and M Reeken, Nonstandard Analysis: Axiomatically, Springer-Verlag Berlin Heidelberg New York, 2004, xvi + 408 pages.

[8] E Nelson, Internal set theory: a new approach to Nonstandard Analysis, Bull. Amer. Math. Soc. 83 (1977), 1165-1198; doi: 10.1090/S0002-9904-1977-14398-X.

[9] Y Péraire, Théorie relative des ensembles internes, Osaka J. Math. 29 (1992), 267-297.

The City College of New York

khrbacek@sci.ccny. cuny . edu

Received: 31 October $2011 \quad$ Revised: 22 March 2012 